\title{
Denial of Service Resilience in Ad Hoc Networks
}

\author{
Imad Aad,${ }^{\dagger}$ Jean-Pierre Hubaux ${ }^{\dagger}$ and Edward W. Knightly ${ }^{\ddagger}{ }^{*}$ \\ ${ }^{\dagger}$ School of Computer and Communication Sciences \\ Swiss Federal Institute of Technology (EPFL) \\ Lausanne, Switzerland \\ \{imad.aad, jean-pierre.hubaux\}@epfl.ch \\ ${ }^{\ddagger}$ ECE/CS Departments \\ Rice University \\ Houston, TX \\ knightlyarice.edu
}

\begin{abstract}
Significant progress has been made towards making ad hoc networks secure and DoS resilient. However, little attention has been focused on quantifying DoS resilience: Do ad hoc networks have sufficiently redundant paths and counter-DoS mechanisms to make DoS attacks largely ineffective? Or are there attack and system factors that can lead to devastating effects? In this paper, we design and study DoS attacks in order to assess the damage that difficultto-detect attackers can cause. The first attack we study, called the JellyFish attack, is targeted against closed-loop flows such as TCP; although protocol compliant, it has devastating effects. The second is the Black Hole attack, which has effects similar to the JellyFish, but on open-loop flows. We quantify via simulations and analytical modeling the scalability of DoS attacks as a function of key performance parameters such as mobility, system size, node density, and counter-DoS strategy. One perhaps surprising result is that such DoS attacks can increase the capacity of ad hoc networks, as they starve multi-hop flows and only allow one-hop communication, a capacity-maximizing, yet clearly undesirable situation.
\end{abstract}

\section{Categories and Subject Descriptors}

C.2.0 [Computer Communication Networks]: General-Security and Protection; C.2.2 [Computer Communication Networks] Network Protocols-Routing protocols; C.2.6 [Computer Communication Networks]: Internetworking—Standards

\section{General Terms}

Performance, Security

\begin{abstract}
*The research of E. Knightly is supported by NSF ITR grants ANI0331620 and ANI-0325971, by a Sloan Fellowship, and by Intel Corporation. The research of I. Aad was partially supported by a postdoctoral fellowship from INRIA. The research of J.P. Hubaux and I. Aad is partially supported by the National Competence Center in Research on Mobile Information and Communication Systems (NCCR-MICS), a center supported by the Swiss National Science Foundation under grant number 500567322 (http://www.terminodes.org).
\end{abstract}

Permission to make digital or hard copies of all or part of this work for personal or classroom use is granted without fee provided that copies are not made or distributed for profi t or commercial advantage and that copies bear this notice and the full citation on the fi rst page. To copy otherwise, to republish, to post on servers or to redistribute to lists, requires prior specifi c permission and/or a fee.

MobiCom'04, Sept. 26-Oct. 1, 2004, Philadelphia, Pennsylvania, USA. Copyright 2004 ACM 1-58113-868-7/04/0009 ...\$5.00.

\section{Keywords}

DoS attacks, TCP, UDP, ad hoc networks

\section{INTRODUCTION}

Significant progress has been made in securing ad hoc networks via the development of secure routing protocols $[2,11,16,17$, 34]. Moreover, ensuring resilience to misbehavior and denial-ofservice attacks has also been the focus of significant research efforts as such resilience is a critical component of a secure system: examples include "watch-dog" mechanisms designed to detect and circumvent misbehaving nodes [27]; rate-limiting of route-request messages to prevent route query-flood attacks [17]; and "rushing attack prevention" that seeks to inhibit malicious nodes from attracting an excessive number of routes, which would increase their ability to inflict damage [20].

Yet, there remains an indefinite "arms race" in system and protocol design: attackers (or researchers anticipating the moves of attackers) will continually introduce increasingly sophisticated attacks, and protocol designers will continually design protocol mechanisms designed to thwart the new attacks.

The goal of this paper is to quantify via analytical models and simulation experiments the damage that a successful attacker can have on the performance of an ad hoc network. In particular, we recognize that successful attacks are inevitable (at least until the corresponding counter-DoS protocol modification is deployed), and our objective is to characterize the relationship between the resources that must be commandeered by the attacker (the percentage of nodes in an ad hoc network used in the attack) and the impact on performance of non-attacking nodes, where performance refers to per-flow goodput and system-wide fairness. In this way, we study the scalability of DoS attacks and identify the key mechanisms and factors of both attacks and protocols that affect a system's DoS resilience.

Our methodology is to study DoS resilience via a new and general class of protocol compliant denial-of-service attacks, which we refer to as JellyFish (JF). Although previously studied attackers disobey protocol rules, JellyFish conform to all routing and forwarding protocol specifications, and moreover, as implied by the name, are passive and difficult to detect until after the "sting." JellyFish target closed-loop flows that are responsive to network conditions such as delay and loss. Examples include TCP flows and congestioncontrolled UDP flows employing a TFRC-like algorithm [13].

The goal of JF nodes is to reduce the goodput of all traversing flows to near-zero while dropping zero or a small fraction of packets. In particular, JF nodes employ one of three mechanisms. The first JF variant is a packet misordering attack. TCP has a wellknown vulnerability to misordered packets due to factors such as route changes or the use of multi-path routing, and a number of 
TCP modifications have been proposed to improve robustness to misordering [3, 4, 33, 35]. However, no TCP variant is robust to malicious and persistent reordering as employed by the JF misordering attack. The second JF mechanism is periodic dropping according to a maliciously chosen period. This attack is inspired by the Shrew attack [25] in which an endpoint sends maliciously spaced periodic pulses in order to force flows into repeated timeout phases [25]. The JF periodic dropping attack utilizes the same principles but realizes the attack via periodic dropping at relay nodes. In particular, suppose that congestion losses force a node to drop $x \%$ of packets. As shown in [25], if these losses occur periodically at the retransmission-time-out timescale (approximately 1 second), TCP throughput is reduced to near zero even for small values of $x$. Thus, a JF periodic-dropping node can drop no more packets than neighboring congested nodes, but inflict near-zero throughput on all TCP flows traversing it. Third, we consider a delay-variance attack in which the attacker randomly delays packets (preserving order) in order to thwart TCP's timers and congestion inferences. This attack not only thwarts widely deployed TCP variants, but also can disrupt rate-based congestion control algorithms such as [5, 9]. Notice that JF nodes are protocol compliant in that IP's datagram service does not mandate loss-free service, in-order delivery, or bounded delay jitter.

Finally, in addition to the JF attack, we also study the "black hole" attack as described in [17]. This attack is relevant for openloop flows that do not respond to congestion, loss, or delay information, and hence cannot be thwarted by JellyFish. Black Hole nodes participate in the routing protocol to establish routes through themselves, yet drop all packets after correctly receiving them at the MAC layer.

With these attacks (three JF variants and Black Holes), we use a combination of analytical modeling and simulation experiments to study the key performance factors that determine a network's DoS resilience and equivalently, the attack's scalability.

Throughout, we consider that victims will diagnose and react to DoS attacks. Thus, we quantify the relationship between the timescale of diagnosis and reaction to the attacker as compared to the route lifetime. Intuitively, if a system has no mobility (and infinite route lifetimes), JF will have little effect as nodes will eventually discover routes without JF if such routes exist. However, as mobility increases, the route lifetime shortens and the effects of JF become increasingly pronounced as the time spent uselessly transmitting on JF paths and re-establishing routes becomes an increasing fraction of a flow's lifetime. Thus, we derive an analytical and experimental relationship that characterizes the impact of these timescales on flow goodput.

Finally, we study a number of system factors that affect a network's DoS resilience and obtain the following findings. (i) JF have a network partitioning effect that severely degrades or altogether prevents long-range communication. Consequently, an increased number of JF reduce the system's fairness index but increase network capacity, as capacity can be increased by starving long-range flows and serving only one-hop flows. (ii) The mean and distribution of path length have a significant effect on attack scalability as higher path length flows are highly vulnerable. (iii) JF are most devastating in a system with a well balanced offered load. If a system is heavily overloaded, system performance is already so poor (high path length flows are already starved), that JF have little marginal impact. (iv) Random or mobile JF placement performs nearly identical to optimal-coverage JF placement in systems with even a small number of JF. (v) JF are most effective in moderate to high density networks as excessively low density networks may already be partitioned and JF can do little marginal damage. (vi)
The scaling of the attack with the percentage of JF remains largely unaffected for large vs. small scale networks. Yet, the absolute performance is quite different, as without attack, small scale network performance is significantly better than large scale network performance.

Thus, our goal is not to advance the aforementioned "arms race" by developing attacks, victim counter strategies, counter attacks, etc., but rather to explore the impact of a class of attacks that are difficult and time consuming to detect due to their compliance to all protocol rules. Yet, we do consider that bad paths will indeed be diagnosed by victims and routed around (as will be the case with the JF attack or other yet-to-be-invented attacks) and we study the key performance factors for attack scalability.

The remainder of this paper is organized as follows. In Section 2 we present the JF attacks and an example of their effects on throughput. In Section 3 we present a simple analytical model that relates system properties such as mean-path-duration and meanpath-length to the victim's throughput. In Section 4 we perform extensive simulation experiments to quantify the factors that control an attack's scalability. Finally, in Section 5 we review related work and in Section 6 we conclude.

\section{JELLYFISH AND BLACK HOLE DOS ATTACKS}

\subsection{System Model}

Unless otherwise specified, we consider a general mobile ad hoc network employing a broad set of security and DoS resilience mechanisms that (i) ensure node authentication, (ii) ensure message authentication, (iii) ensure one identity per node (preventing Sybil attacks), and (iv) prevent control plane misbehavior (query floods, rushing attacks, etc.).

Examples of protocols that achieve the above objectives are discussed in Section 5, but for concreteness, we can consider a secure source routing protocol as in reference [17] as well as enhancements such as $[19,20]$. Throughout the paper and especially in Section 3, we discuss the implications of such enhancements, as well as other counter DoS mechanisms. However, we do not consider deployment of reputation mechanisms, yet discuss such protocols in Section 5.

The effects of the DoS attacks we describe are independent of the considered MAC layer protocol. However, in our simulations, we consider the MAC layer to be IEEE 802.11.

A fraction of nodes are malicious and seek to thwart system performance. A malicious node will always participate in route setup operations. For example, if source routing is employed, malicious nodes always relay Route Request packets in order to have as many routes as possible flowing through themselves; if distance vector routing is employed, malicious nodes will also obey all controlplane protocol specifications. However, once a route is established, attacking nodes will thwart the end-to-end throughput of the flow via a JellyFish or Black Hole attack. While packets may be encrypted at higher layers and become "unrecognizable" (e.g., TCP vs. UDP) to the network layer, the JellyFish and Black Hole attacks can still be applied irrespective of the packet types.

\subsection{JellyFish Attack}

A critical strength of the JellyFish Attack is that it maintains compliance with all control plane and data plane protocols in order to make detection and diagnosis costly and time consuming. The key principle that JF use to facilitate the attack is targeting end-toend congestion control. In particular, many applications such as 
file transfer, messaging, and web will require reliable, congestioncontrolled delivery as provided by protocols such as TCP. Moreover, TFRC-controlled real-time applications such as interactive video must also adapt their rates to available bandwidth and hence also employ end-to-end congestion control.

The dual role of hosts as routers in ad hoc networks introduces a critical vulnerability for congestion control: specifically, there are a number of forwarding behaviors that routers (ad hoc relay nodes) can employ that will severely degrade the end-to-end throughput of congestion-controlled traffic. We refer to these behaviors as variants of the JellyFish attack, which we describe as follows.

JF Reorder Attack. TCP's use of cumulative acknowledgements defines the message "ACK- $N$ " to indicate that all segments $1, \cdots, N$ have been received. Consequently, receipt of duplicate ACKs is used to infer loss. Yet, because duplicate ACKs can also indicate an out-of-order packet receipt, TCP has a number of mechanisms to increase its robustness to out-of-order packets, including TCP Sack [12] and reorder robust TCP [35]. Yet, all such TCP variants assume that reordering events are rare, short-lived, and due to network events such as route changes.

In contrast, we consider JF nodes to maliciously re-order packets. In this attack, JF deliver all packets, yet after placing them in a re-ordering buffer rather than a FIFO buffer. Consequently, we will show that such persistent re-ordering of packets will result in near zero goodput, despite having all transmitted packets delivered.

JF Periodic Dropping Attack. Losses due to buffer overflow are inevitable in congested environments. Kuzmanovic and Knightly [25] show that if such losses occur periodically near the retransmission time out (RTO) timescale (in the 1s range as RTO is intended to address severe congestion), then end-to-end throughput is nearly zero. An endpoint attack is described in [25] in which a malicious node transmits periodic pulses into the network. As the RTO-spaced pulses can force all flows sharing the bottleneck link to enter repeated timeout phases, the attack results in all such flows obtaining near-zero throughput while the attacker has a low average transmission rate. The study showed that the impact of the attack can be quite severe whether minimum RTO values are all set to 1 second as recommended in [31], or are randomized over a wide range.

Here, we utilize the same principle for the JF periodic dropping attack in which attacking nodes drop all packets for a short duration (e.g., tens of ms) once per RTO. Thus, unlike [25], JF are passive and generate no traffic themselves; like non-malicious nodes, JF drop for only a small fraction of time; yet, with this dropping pattern during a maliciously chosen period, the following behavior results. Upon encountering the JF's first loss duration, the victim flow will enter timeout as the JF chooses the dropping duration to be sufficiently long to result in multiple losses. When the flow attempts to exit timeout RTO seconds later, the JF will immediately or soon after periodically drop again. Note that the JF knows when a flow enters timeout as the JF itself induced the loss. Thus, the JF can safely assume that by RTO seconds later, the flow will be attempting to exit and will be in the fragile slow-start state.

JF Delay Variance Attack. Variable round-trip-times due to congestion are an inevitable component of TCP's operation. Yet, ensuring high performance in the presence of random and high delay variation due to an attacker was clearly not incorporated into TCP's design. Such a high delay variation can (i) cause TCP to send traffic in bursts due to "self-clocking," leading to increased collisions and loss, (ii) cause mis-estimations of available bandwidth for delay-based congestion control protocols such as TCP Westwood and Vegas, and (iii) lead to an excessively high RTO value.
Indeed, enhancing TCP to combat the effects of non-malicious delay variation to wireless links has been the focus of intense research (see [10] for example), as has the development of tools for available bandwidth estimation. Consequently, malicious manipulation of packet delays by the JF delay variance attack has the potential to significantly reduce TCP throughput. Such attackers therefore wait a random time before servicing each packet, maintaining FIFO order, but significantly increasing delay variance.

\subsection{Impact of JF}

We next present simulation experiments that illustrate the effects of JF on end-to-end goodput. To study these effects in isolation, we consider a simple "chain" scenario with a sequence of nodes between the sender and receiver, one of which is a JF. We use the default IEEE 802.11 MAC at $2 \mathrm{Mb} / \mathrm{s}$ and TCP Sack.

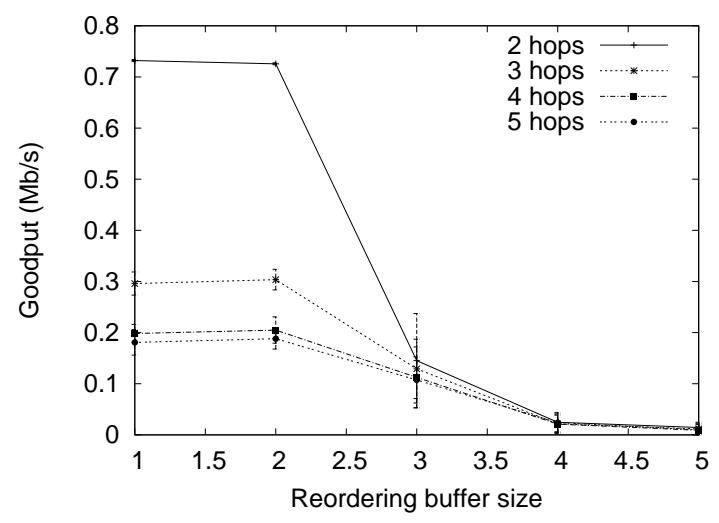

Figure 1: JF-reorder effect on throughput

Figure 1 shows the impact of the JF-reorder attack on the TCPSack flow for different re-ordering. This experiment has a scheduler that is a FIFO queue, except that it selects randomly among the first $k$ packets in the queue. The figure depicts performance as a function of the re-ordering buffer size expressed in packets.

The figure indicates that TCP is robust to moderate reordering with a reordering buffer of 2 packets. Whereas, when the reordering buffer is larger and the reordering is performed in this persistent and malicious way, TCP throughput collapses. For example, consider the curve with 3 nodes and a 2-hop chain, i.e., a source, destination, and a single relay node. Without an attack (a reordering buffer of 1), the flow obtains a throughput of $710 \mathrm{~kb} / \mathrm{s}$. Yet, with a reordering buffer of 3 or more packets, the throughput decreases to approximately $1 \%$ of the peak value indicating a successful attack and near starvation of the flow. That is, if the scheduler selects the next packet to service randomly among the first 3 or more queued, the resulting reordering cannot be overcome by TCP. We note that solutions to TCP reordering such as TCP-PR [4] use only timers to detect loss vs. duplicate ACKs. Thus, attackers would need to either use other JF variants for TCP-PR flows or use larger reorder buffers to force TCP-PR timeouts.

Figure 2 depicts the results of simulation experiments with the JF periodic dropping attack. Consider first the upper curve in which the path consists of a source, a single relay node (a JF), and a destination. A time period of 0 indicates no attack and the flow again obtains a throughput of $710 \mathrm{~kb} / \mathrm{s}$. As in [25], the degradation in throughput to the victim is highly non-linear as a function of the dropping period, with null frequencies near 0.5 and 1 second (the minimum RTO value). To obtain the null at 1 second, the JF drops 
packets for $90 \mathrm{~ms}$ every 1 second, which results in dropping $9 \%$ of the time, and forwarding $91 \%$ percent of the time, values easily incurred by a congested node.

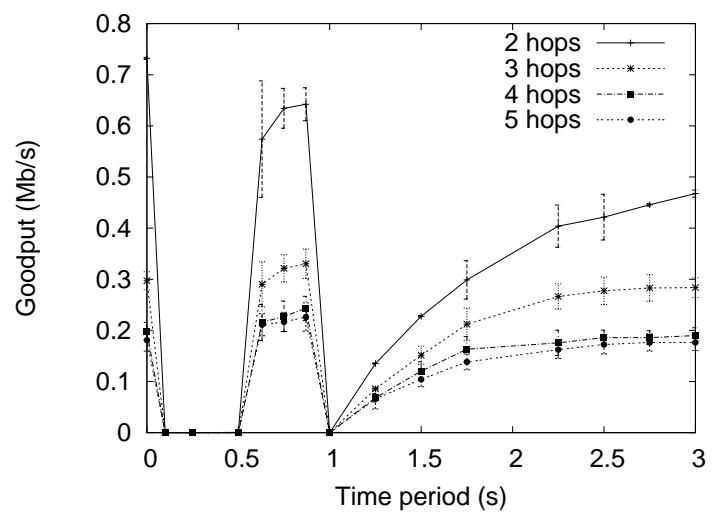

Figure 2: JF-drop effect on throughput

The attack is therefore successfully exploiting the slow-timescale congestion avoidance mechanism of TCP, namely, that flows must infer that multiple packet losses within a round-trip-time are an indication of severe congestion, such that the flow must back off aggressively, and wait RTO seconds before entering slow start. Significantly reducing RTO or removing the mechanism all together would lead to significant spurious retransmissions and potentially congestion collapse [31], whereas increasing the value would make the attack even more devastating.

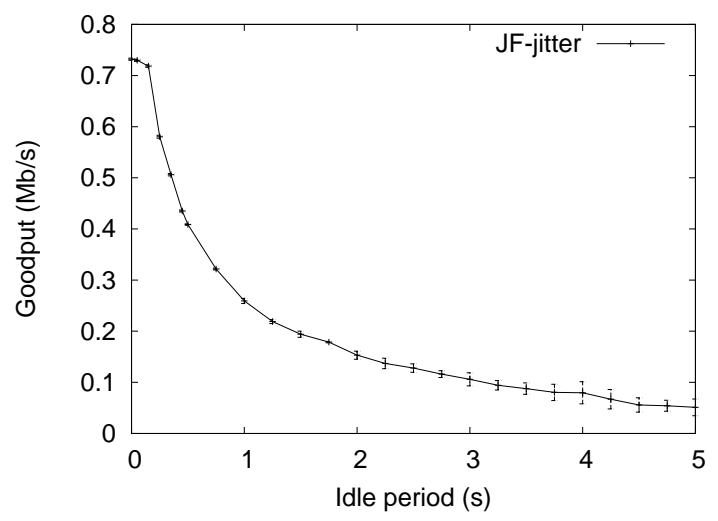

Figure 3: JF-jitter effect on throughput

Finally, we performed experiments with the JF delay variance attack in a three node chain. In this scenario, the JF behaves as a server with vacations, alternating between periods of serving no packets (and queueing, but not dropping them) and serving packets at its maximum capacity. Both idle and active periods are of equal lengths. Figure 3 shows how TCP goodput decreases with increasing jitter (i.e., increasing idle and active periods). While this descreased throughput is also due to increased mean delay (i.e., mean RTT), the figure none-the-less indicates that the effects of this attack can be quite severe.

\subsection{Black Hole Attacks}

We also consider Black Hole attacks as described in [17]. As with JF, Black Hole nodes participate in all routing control plane operations. However, once paths are established, Black Holes simply drop all packets. Although refusing to forward data is not protocol compliant, we also study Black Holes for the following reasons. First, as demonstrated in the simulations above, JF have nearly the same impact as Black Holes, making end-to-end throughput collapse until the victim detects and fixes the problem. Thus, in many simulation experiments, we will consider Black Holes in place of JF for simplicity. Second, Black Holes allow us to study flows that are not congestion controlled and therefore are immune to JF. Thus, we can still study attack scalability for open-loop flows that ignore the delay, ordering, and loss information that JF are manipulating.

\subsection{Misbehavior Diagnosis}

Victims of the attacks will measure that they have near zero endto-end throughput and will react. Likewise, a malicious node's neighbors may attempt to diagnose failed paths due to DoS behavior. Clearly, the attacker seeks to inhibit diagnosis in order to maximize damage. Thus, we next explore network and endpoint mechanisms for DoS diagnosis with a focus on their practical feasibility, as well as on the timescales for successful diagnosis and repair. In Section 3 we quantify the effects of these timescales on flow goodput and in Section 4 we experimentally explore this factor.

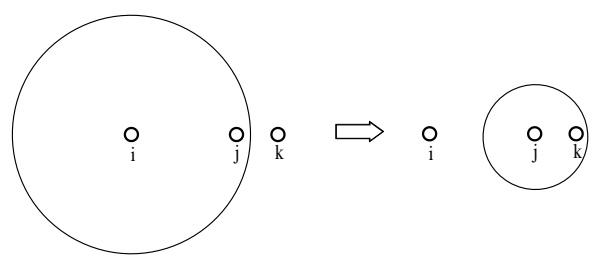

(a) Energy Efficient Transmission: $i$ cannot overhear $j$

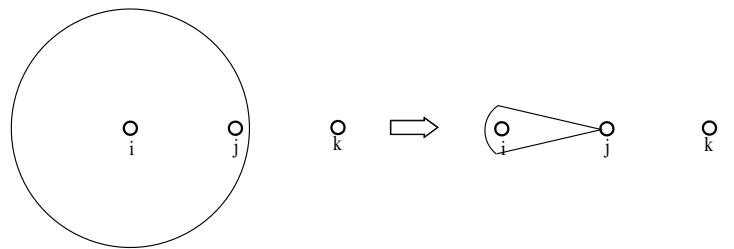

(b) Directional Antennas: $j$ pretends to $i$ to forward to $k$

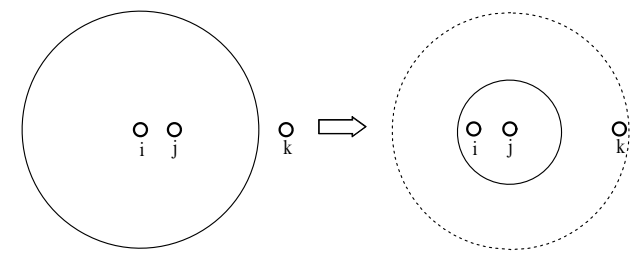

(c) Variable Power: $j$ pretends to $i$ to forward to $k$

Figure 4: PACK Limitations

Detection of MAC Layer Failure. Because mobility can cause routes to break, routing protocols have mechanisms to detect broken routes. For example, DSR uses a MAC layer transmission failure (indicated by multiple transmission attempts that fail to generate an acknowledgement) to generate a route error message. This message is sent upstream to the source node, which will subsequently establish a new route. But this will not occur in the 
JellyFish nor Black Hole attack as such DoS nodes are protocol compliant at the MAC layer.

Passive Acknowledgement (PACK). Consider the Black Hole attack in which a malicious node drops all packets. The malicious node must first correctly receive and acknowledge the packet at the MAC layer (as described above) in order to prevent the upstream neighbor from immediately diagnosing a broken route. Yet, if the Black Hole node fails to forward the packet, can this be detected by eavesdropping neighbors?

Passive Acknowledgements (PACK) [23] as well as "watchdog" protocols [27] were designed precisely for this purpose. The key idea of PACK is to exploit the broadcast nature of the wireless medium: if node $i$ sends a packet to $k$ via $j$ (Fig. 4(a)), then $i$ should overhear the subsequent transmission from (neighboring) $j$ to $k$.

Unfortunately, PACK has three key limitations that preclude its use as a general solution to such attacks. First, PACK cannot be deployed in combination with dynamic power management. In particular, continuing with the above example, PACK requires that node $j$ 's transmissions to $k$ must be overheard by node $i$. This in turn implies that $j$ cannot reduce its power level when transmitting to $k$, even if the path from $j$ to $k$ has low distance or high SNR. Consequently, precluding the use of dynamic power management (in order to deploy PACK) not only results in a significant waste of energy but also inhibits spatial reuse.

Such a scenario is illustrated in Figure 4(a). Here, nodes $j$ and $k$ are closer than nodes $i$ and $j$. However, if node $j$ employs dynamic power management techniques (as in [24] for example) and reduces its transmission energy when communicating to node $k$, then node $i$ will incorrectly infer that node $j$ is misbehaving and will take action according to the specific routing protocol and counter-DoS technique. Second, PACK and watchdogs implicitly assume that attackers will use omnidirectional antennas. If a Black Hole node uses instead a directional antenna (electronically steerable or sectorized), then it can fool its upstream node by beam-forming as illustrated in Figure 4(b). In particular, the Black Hole node will beam-form a fake $j$ to $k$ transmission such that $i$ incorrectly infers that $k$ has received the packet. ${ }^{1}$ This issue will be increasingly critical as such technologies become widely deployed. Third, as illustrated in Figure 4(c), a node can also use variable power transmission to thwart PACK. In the figure, $i$ is closer to $j$ than $j$ is to $k$ so that $j$ can pretend to $i$ to have forwarded the packet, yet $j$ has reduced its power such that only $i$ and not $k$ can receive it.

In any case, because JellyFish attacks are protocol compliant, it will be quite problematic for a neighbor to detect their misbehavior. For example, the JF periodic dropping attack can drop packets at a rate no higher than that of a congested node. Moreover, even behaving nodes can drop packets in bursts due to the correlation of a queue state (a full buffer leads to all arriving packets being dropped until a transmission). Likewise, a high variance in queueing delay may be impossible for observing neighbors to distinguish from behavior that results from fading channels and variable rate traffic. Whereas the JF-reorder attack may be the easiest JF variant to detect via a PACK-like mechanism. Yet, to detect this attack, IP would need to be changed to mandate in-order delivery, and the above problems with PACK would need to be solved.

\footnotetext{
${ }^{1}$ Clearly, if node $i$ anticipates attackers with directional antennas, it could then demand a signed acknowledgement from $k$. Yet, we do not further study the overhead and performance implications of such escalations in the "arms race" for the reasons described in Section 1 .
}

Layer 4 Endpoint Detection. As relying on neighbors to detect JF and even Black Holes is quite problematic for the reasons outlined above, attack victims will need to rely on end-to-end mechanisms. The key tradeoff for endpoints will be balancing fast detection with false positives. At one extreme, endpoints could use a single packet loss (indicated by a timeout) as a fast indication of a problematic route. Yet, reacting to the first loss (via a new route request, for example) would necessarily lead to many false inferences of attack, as congestion, fading channels, etc. can also lead to loss and timeout.

At the other extreme, endpoints could require a large number of packets to timeout before inferring that the path has malfunctioned. In this case, nodes would indeed avoid spurious reactions to congestion. Yet when attackers are truly present, victims would spend a larger fraction of time before establishing a new successful path. Thus, endpoints must devise a policy that balances between these two extremes.

Therefore, a key performance factor of DoS resilience is the diagnosis time scale. Given that end-to-end mechanisms are the only viable solution for attack detection, and that the minimum time for timeout recommended for even a single congestion-induced loss is one second [31], we expect detection timescales to be on the order of several seconds. In any case, we explore a range of values for this timescale throughout the paper.

\subsection{Victim Response}

Once a path is diagnosed as providing zero throughput, the end points will attempt to establish an alternate path. With uni-path source routing, this will be achieved via transmission of a new route route request message, typically from the source. When route replies are received, the victim should avoid paths with any node from the prior malfunctioning path as the victim does not know which node on the path was malicious, i.e., the victim has insufficient information to form an accurate "black list." Furthermore, note that as JF are protocol compliant, the victim is not certain whether throughput collapsed due to an attacker or simply due to congestion, fading, or other factors incurred in normal protocol operation.

An alternate solution is to employ multipath routing, and to adapt the path weights according to path goodput as proposed in $[17,30]$. Even without attackers, such a protocol must overcome the impact of different paths having different delay characteristics and the corresponding impact on TCP throughput. For example, reference [1, 14] found that TCP Sack's use of multiple paths in ad hoc networks led to a severe throughput reduction for even two paths, and near collapse for three or more paths. The authors then suggest a redesign of TCP to support multipath routing.

Other promising counter-measures would be the establishment of backup routes, e.g., caching of all route reply messages for later use if a current path fails.

In any case, even with multipath routing and TCP re-design, use of backup routes, etc., a victim flow will always encounter the issues we study next: delays to diagnose and react to the problem, and poor throughput until all forwarding paths are free of JF.

\section{ANALYTICAL MODEL}

In this section, we develop a simple model to predict the throughput of a flow traversing a network in the presence of attacking nodes.

Consider an ad hoc network with $N$ nodes and $a<N$ attacking nodes (JellyFish or Black Holes). Denote $p$ as the probability that a randomly selected node is an attacker such that $p=a / N$. (We also discuss other relationships between $a, N$, and $p$ below.) Consider 
a path traversing $h$ relay hops. If the selected nodes represent a random sample of the $N$ network nodes, then the path contains no attacking nodes with probability $(1-p)^{h}$.

We compute the throughput via a renewal argument in which time alternates between periods of successful transmission and periods of thwarted transmissions and assume that such durations are independent and identically distributed. In particular, we denote $E\left(T_{L}\right)$ as the expected lifetime of a route as determined by factors such as the node velocity and node density.

When a route breaks due to mobility, a number of delays are incurred in repairing the route. First, a duration $T_{\text {diag }}$ is incurred to diagnose that the route is broken. Next, the request for a new route may be delayed by a rate-limiting duration in order to mitigate the impact of route query flood attacks. We denote this rate-limiting time as $T_{R L}$, which denotes the minimum inter-spacing of route requests allowed by the routing protocol. Finally, the node must wait to receive one or more route reply messages, a duration that we denote as $T_{R R}$.

After these three phases, a node begins transmitting data on the new path. However, the new path includes at least one attacking node with probability $1-(1-p)^{h}$. If this is the case, the transmission is thwarted and the node must again incur the above three delays and try again. Note that even if the victim has ensured that the new route contains no nodes in common with a failed route, the new route may again contain an attacking node. Thus, a node exits the zero-throughput phase only after it has successfully established a route without an attacking node.

In general, a protocol may change timers according to the number of attempts. Thus we denote superscript $n$ as the attempt number such that for example $T_{R L}^{n}$ denotes the rate-limiting duration waited immediately before the $n^{t h}$ attempt. Thus, we have that the total expected time of zero throughput, i.e., the time to find a new route that contains no attacking node, is given by

$$
\begin{gathered}
E\left(T_{0}\right)=\sum_{n=1}^{\infty}\left(\sum_{j=1}^{n} E\left(T_{\text {diag }}^{j}\right)+\sum_{j=1}^{n} E\left(T_{R L}^{j}\right)+\sum_{j=1}^{n} E\left(T_{R R}^{j}\right)\right) \\
(1-p)^{h}\left(1-(1-p)^{h}\right)^{n-1} .
\end{gathered}
$$

More generally, the path length can be represented by a random variable $H$ such that $E\left(T_{0}\right)=\sum_{h>0} E\left(T_{0} \mid H\right) \operatorname{Pr}(H=h)$ using Equation (1) for $E\left(T_{0} \mid H\right)$ along with the distribution of $H$. To simplify, we consider a fixed path length $(H=h)$ unless otherwise noted, and consider the further simplification $E\left(T^{i}\right)=E\left(T^{j}\right)$ such that we have

$$
\begin{array}{r}
E\left(T_{0}\right)=\sum_{n=1}^{\infty} n\left(E\left(T_{\text {diag }}\right)+E\left(T_{R L}\right)+E\left(T_{R R}\right)\right) \\
(1-p)^{h}\left(1-(1-p)^{h}\right)^{n-1} .
\end{array}
$$

The normalized goodput for a flow is given by

$$
\frac{E\left(T_{L}\right)}{E\left(T_{L}\right)+E\left(T_{0}\right)}
$$

which under the above assumptions reduces to

$$
\frac{E\left(T_{L}\right)}{E\left(T_{L}\right)+\left(E\left(T_{\text {diag }}^{n}\right)+E\left(T_{R L}^{n}\right)+E\left(T_{R R}^{n}\right)\right)(1-p)^{-h}} .
$$

We make several observations about Equation (4). First, note the corner case with $p$ approaching 1 or high route length send goodput to 0 . Another corner case is a scenario with no mobility: in this case, once a successful route is established, it is never subsequently broken and goodput approaches 1 .

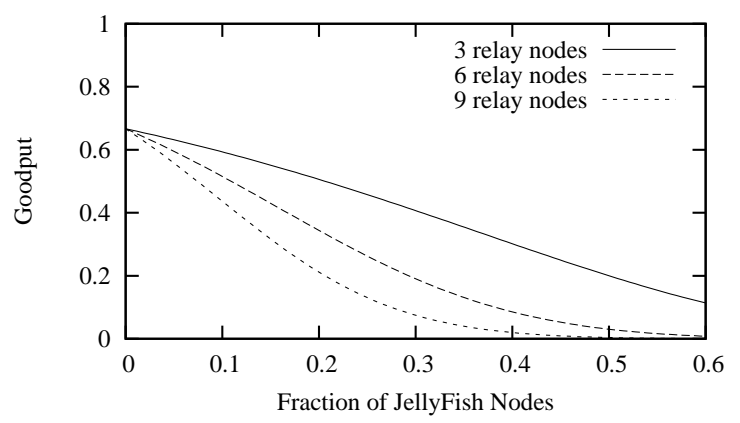

Figure 5: Attack Scalability and Path Length

Intermediate cases are depicted in Figure 5, which illustrates goodput (computed from Expression (4)) as a function of the percentage of attackers $p=a / N$ for three route lengths of 3,6, and 9 relay nodes. We consider a mean route lifetime of $E\left(T_{L}\right)=10$ $\mathrm{s}$, which corresponds to a high node velocity of $\mathrm{Vmax}=30 \mathrm{~m} / \mathrm{s}$ as reported in [32]. Moreover, the curves depict the case that the diagnosis, rate limit, and route reply times are $2 \mathrm{~s}, 2 \mathrm{~s}$, and $1 \mathrm{~s}$ respectively. The diagnosis time is set to two times the default retransmission timeout value for TCP: a lower value would certainly lead to false inference of broken routes. The 2 second rate limiter value is the default value for DSR for the minimum spacing of route requests, which is increased in DSR to 10 seconds for subsequent requests (not shown here).

The figure indicates that without any attacking node, legitimate nodes spend approximately $66 \%$ of their time successfully transmitting, and the remaining $33 \%$ having broken routes and trying to re-establish routes due to mobility. Note that even though nodes can retransmit packets lost during this time, or even delay transmission during phases of broken routes, this result indicates that the flows will obtain $66 \%$ of the throughput that they would have obtained in a static scenario without mobility. Next observe the scalability of the attack for 6 relay nodes: with $10 \%$ of attacking nodes, the goodput drops to $52 \%$, whereas with $20 \%$ of attacking nodes, the goodput drops to $34 \%$. The impact of the attacker is even more pronounced in large-scale networks in which a longer path length is increasingly likely to include an attacking node. For example, with 9 relay nodes, the goodput decreases to $44 \%$ under $10 \%$ attacking nodes and to $21 \%$ under $20 \%$ attacking nodes.

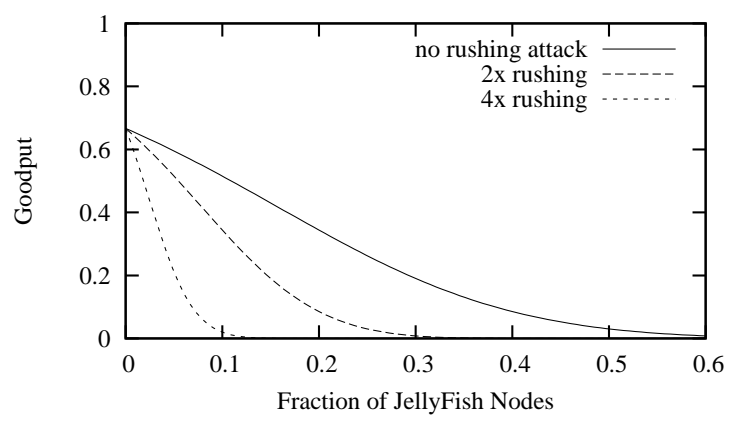

Figure 6: Impact of the Rushing Attack

The model also allows us to explore the impact of a "Rushing Attack" as described in [20]. In rushing attacks, malicious nodes 
use different mechanisms to attract flows to route through them, thereby increasing the damage they can do during attack. For example, suppose an attacking node could transmit at (and receive from) twice the distance of other nodes. In such a case, this node would attract a disproportionate number of flows to route through itself as the "shortest path route" would, with a high probability, go through this long-distance hop. Figure 6 characterizes the effect of an attacking node being able to attract two and four times the number of flows due to a rushing attack for path lengths of 6 relay nodes. In this case, the probability of a flow not being intercepted by an attacking node is decreased to $(1-2 p)^{h}$ and $(1-4 p)^{h}$ respectively.

The figure indicates that when the rushing attack is combined with either of our two DoS attacks, the impact can be quite devastating. For example, if attacking nodes are able to attract twice as many flows as compared to that achieved in a uniform graph (i.e., $2 a / N$ vs. $a / N)$, the flow goodput drops from $52 \%$ to $34 \%$ with $10 \%$ of attackers. Furthermore, if attacking nodes can attract four times as many flows, the goodput nearly collapses to $8.5 \%$ under $10 \%$ of attackers. Thus, in addition to quantifying the impact of rushing attacks, this result illustrates the critical importance of preventing rushing attacks. As a number of techniques were proposed in [20] to thwart such misbehavior, we do not consider it further, and in all experiments we do not give attacking nodes any advantage in attracting routes.

\section{ASSESSMENT OF PERFORMANCE UNDER DOS ATTACK}

In this section, we perform an extensive set of simulation experiments to quantify the impact of DoS attackers on performance and to identify the key factors that determine an attack's scalability. After describing our methodology, we establish a baseline case and then isolate the impact of each factor.

\subsection{Methodology}

Attackers affect performance in a number of ways. The performance metrics below allow us to evaluate the impact of JF on individual flows, as well as on the whole system performance.

- System fairness: To measure fairness, we use Jain's fairness index computed using long-term throughput averages and given by [21]:

$$
F_{J}=\frac{\left(\sum_{i=1}^{m} \gamma_{i}\right)^{2}}{m \sum_{i=1}^{m} \gamma_{i}^{2}}
$$

where $m$ is the total number of flows and $\gamma_{i}$ is the proportion of received packets of flow $i$ during the simulation time. $F_{J}$ is equal to 1 when all flows equally share the network, and is equal to $1 / m$ when a single flow monopolizes all resources (in which case $F_{J} \rightarrow 0$ when $m \rightarrow \infty$ ).

- Number of hops for received packets: We consider random topologies with random traffic matrices. However, JF and Black Holes can have the effect of starving multihop flows and giving all the capacity to one-hop flows that (by definition) have no relay nodes and hence do not encounter JF. This performance measure captures this effect and also characterizes network partitioning in which multihop communication becomes impossible.

- Total system throughput: This measure characterizes the received throughput aggregated over all network flows. Providing all capacity to one-hop flows and starving others can be the capacity-maximizing allocation of bandwidth to flows. Thus, JF and Black Holes often increase total system throughput.

- Probability of interception: This characterizes the probability that a flow encounters a JF in its path. This probability depends on many factors such as the placement of JF, the traffic patterns, the percentage of JF etc. Moreover, all the previously mentioned performance metrics depend on this probability.

Experimental and simulation results showed that delays and jitters in ad hoc networks vary considerably. Therefore they provide no relevant information to be considered in our analysis.

An attack's effectiveness is a function of a number of system parameters. We consider the offered load, the congestion control protocol, the JF placement strategy, node mobility, and node density. We next assess the effect of these parameters on the performance metrics described above by varying them one at a time. We use $n s-2$ simulations and present results averaged over 50 simulation runs, using 18 different topologies / mobility scenarios (8000 simulations in total). We show the corresponding $95 \%$ confidence intervals. Each simulation is $500 \mathrm{~s}$, and results are obtained after a warmup period of 100 seconds. Unless otherwise specified, we use Black Holes to emulate the effects of JellyFish on TCP, as the latter were shown in Section 2 to result in near-zero throughput, resulting in a near identical effect as Black Holes. Moreover, JF can have a slightly stronger effect: for example, with JF, re-ordered and delayed packets are still transmitted end-to-end, consuming additional capacity while not contributing to goodput. To simplify the presentation, we designate the attacking nodes as JF throughout the section; in practice, however, they are Black Hole nodes in the case of UDP flows and JellyFish nodes in case of TCP flows. Degraded channel conditions (e.g. noise, fading etc.) are harmful components to the system performance. Therefore we consider a clear non-fading channel to assess the impact of the JF attacks.

\subsection{Baseline}

For the baseline simulations, we consider a scenario in which 200 nodes move randomly in a $2000 \mathrm{~m} \times 2000 \mathrm{~m}$ topology, at a maximum velocity of $10 \mathrm{~m} / \mathrm{s}$, pausing for $10 \mathrm{~s}$ on average. Nodes use the IEEE $802.11 \mathrm{MAC}$ with a node receive range of $250 \mathrm{~m}$. The channel capacity is $1 \mathrm{Mb} / \mathrm{s}$. 100 of these nodes communicate with each other to create 50 flows. UDP packets are transmitted at a constant rate of $800 \mathrm{bits} / \mathrm{s}$, corresponding to one 500 byte packet every $5 \mathrm{~s}$. The other 100 nodes route packets without generating any flows, and are henceforth called "routers." JF are compromised routers among these 100 . For the baseline, JF are statically placed on a grid as shown in Fig. 7.

Figure 8 shows that in the absence of JF, one-hop flows account for approximately $8 \%$ of received packets, with the remaining packets nearly uniformly allocated to flows up to 5 hops, and then longer-path-length flows accounting for significantly less.

(Note that there is a smaller number of flows having very long paths due to the random traffic matrix.)

However, with $25 \mathrm{JF}$ (12.5\% of nodes), the percentage of received packets corresponding to one-hop flows increases to $20 \%$, and with $49 \mathrm{JF}$ ( $25 \%$ of nodes), the percentage increases to $33 \%$. In each case, this advantage to one-hop flows comes at the cost of multihop flows. For example, under $25 \mathrm{JF}, 5$ hop flows have their throughput cut in half and 10 hop flows become nearly starved. This indicates that the attack has nearly prohibited long-range communication such that the network is in effect partitioned, allowing only short-range communication. 


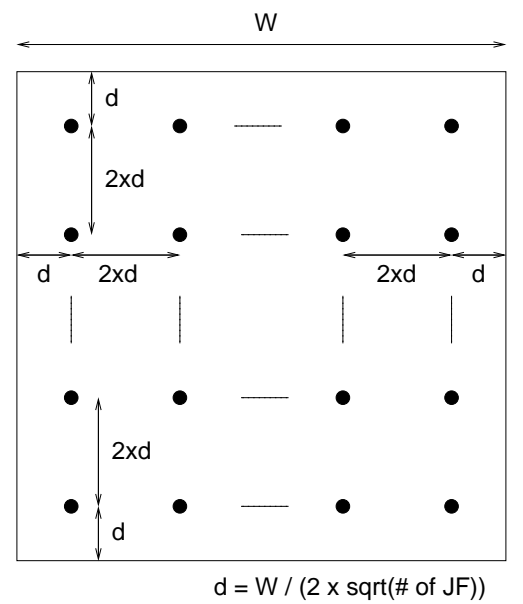

Figure 7: JF placement in a grid

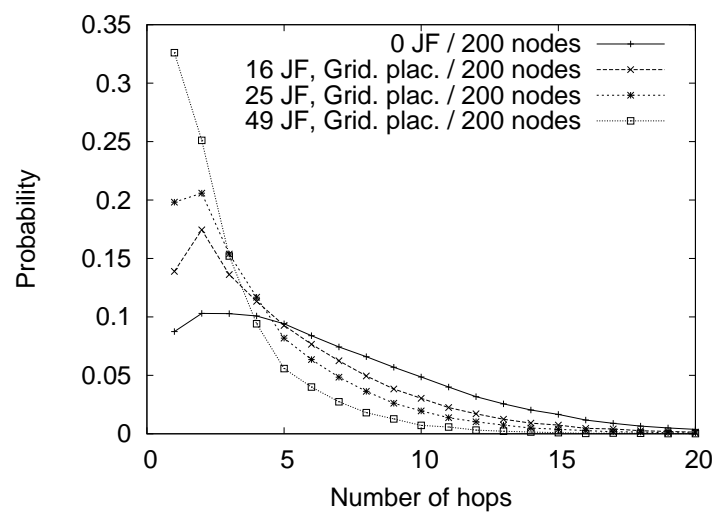

Figure 8: Distribution of the number of hops for received packets

Figure 9 shows the impact of JF on system fairness. Observe that with no JF, the system has a relatively high fairness index of 0.9 indicating that flow rates are not significantly different. However, with an increasing proportion of JF in the network, the fairness index significantly decreases, indicating that some flows are obtaining a significantly higher throughput share at the expense of other flows.

Figure 10 explains the phenomenon. The figure depicts the mean hop length for a received packet. Without attack, the mean is 6.6 indicating that a significant number of packets are received on longpath-length routes. Yet, as the number of JF grows, the average path length for a received packet diminishes: fewer and fewer packets are able to traverse long routes leading to increased capacity for one-hop flows. Figure 8 illustrates the unfairness: long paths are increasingly likely to be intercepted by JF, considerably reducing their share of the system capacity, whereas the short-path flows "benefit" from the attack.

\subsection{Offered Load and TCP}

The system's offered load is an important factor for the scalability and impact of the JF attack. At one extreme, if the offered load is very high, most packets received end-to-end will be over one hop flows even without the attack, so that JF can do little if any addi-

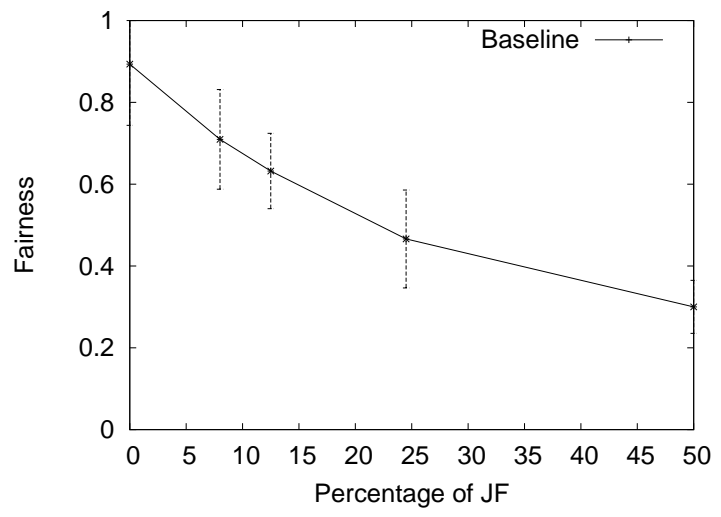

Figure 9: Fairness Index for the baseline case

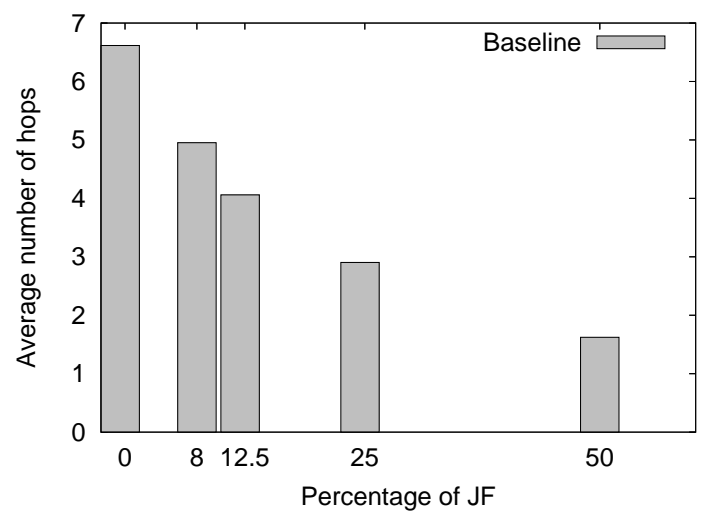

Figure 10: Average number of hops for received packets

tional damage. At the other extreme, with a more moderate load, $\mathrm{JF}$ will skew the distribution of received traffic more towards that achieved in an over-load case.

To study this effect, we consider an offered load per flow of 5 times that of the baseline. Moreover, we consider the offered load that TCP will achieve for 5 and 50 TCP flows. The rest of the parameters remain the same as in the baseline scenario.

The curve in Figure 11 with an offered load of 5 times that of the baseline case illustrates that an overloaded network has a fairness index of 0.4 without any JF, even below that obtained under the baseline load with $25 \% \mathrm{JF}$. Thus, there are too few multihop flows for the JF to even slightly degrade the fairness index, i.e., repeated collisions and buffer overflow severely impede multihop traffic.

For TCP traffic, TCP congestion control does not attempt to provide equal throughput to all flows (which would achieve a fairness index of 1). Instead, it seeks to provide throughput that is inversely proportional to round-trip-time. However, the situation with 50 TCP flows is quite similar to that of the CBR overload case: the JF have little effect on fairness, as one-hop flows are dominating the percentage of packets received end-to-end, even without the attack.

With 5 TCP flows, Figure 12 indicates that without the attack, $40 \%$ of received packets are from one hop flows whereas with 49 JF, this percentage increases to $69 \%$ of received packets. Thus, the attack increased the number of one-hop packets by $73 \%$, resulting in a significant impediment to multihop traffic. 


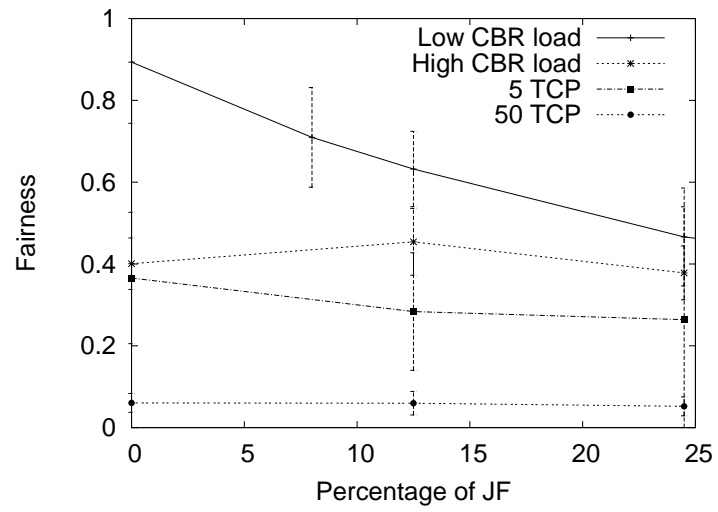

Figure 11: Effect of offered load

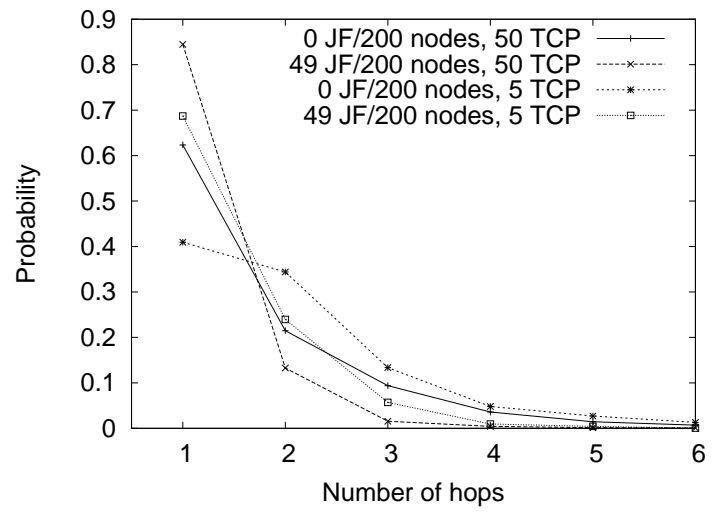

Figure 12: Hops for received packets with different TCP loads

Next we measure the total system throughput as a function of the percentage of JF and present the results in Figure 13. For the baseline case, the figure shows that an increasing percentage of JF results in progressively lower system throughput as an increasingly high number of flows become thwarted by the attack for the reasons discussed above. However, the results are quite different under $5 \mathrm{x}$ system load and for 50 TCP flows. For the case of 5x system load, the total system throughput has nearly doubled under $12.5 \%$ of attackers when compared to no attackers, thus indicating that a DoS attack can increase the capacity of an ad hoc network. Although initially surprising (at least to the authors), the reason is quite simple: JF prevent multi-hop communications, thus liberating significant capacity, which is used by one-hop flows. Thus, Figure 13 shows how misleading capacity can be to express the impact of DoS: communication still continues to take place, but only with one hop neighbors.

We also observe that even under high loads, the behavior is nonmonotonic. The reason is that the existence of surviving flows depends on the topology and node movements: if JF happen to stop a flow that potentially interferes with others, the overall throughput will increase. Otherwise, system throughput is reduced by the thwarted flow's throughput. This dependency on the topology and movement makes the confidence intervals ${ }^{2}$ very large, in spite of averaging over 18 different mobility scenarios of 50 runs each.

\footnotetext{
${ }^{2}$ Not shown, for clarity.
}

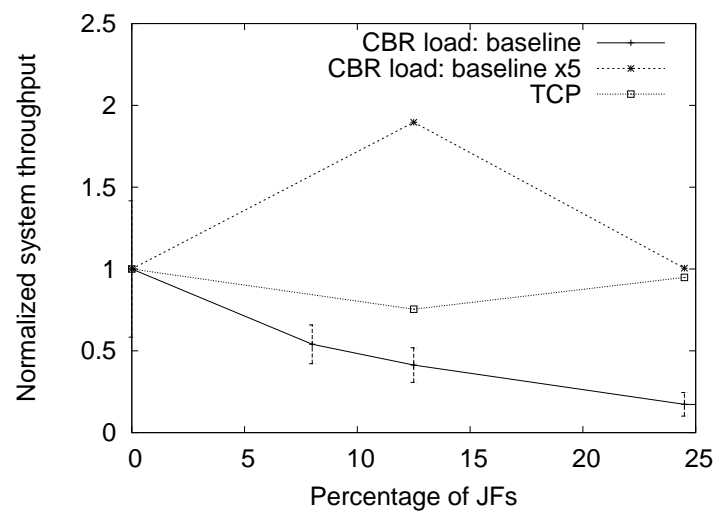

Figure 13: Normalized system throughput with different loads

Thus, with the given topology dimensions of $2000 \mathrm{~m} \times 2000 \mathrm{~m}$ and a high offered load, having 200 nodes with a receive range of $250 \mathrm{~m}$ each and a $500 \mathrm{~m}$ interference range, the first JF added will most likely reduce contention and interference, thus increasing system throughput. But beyond a certain number of JF, no flows can take advantage of this removal of interfering flows anymore, and the system throughput starts decreasing.

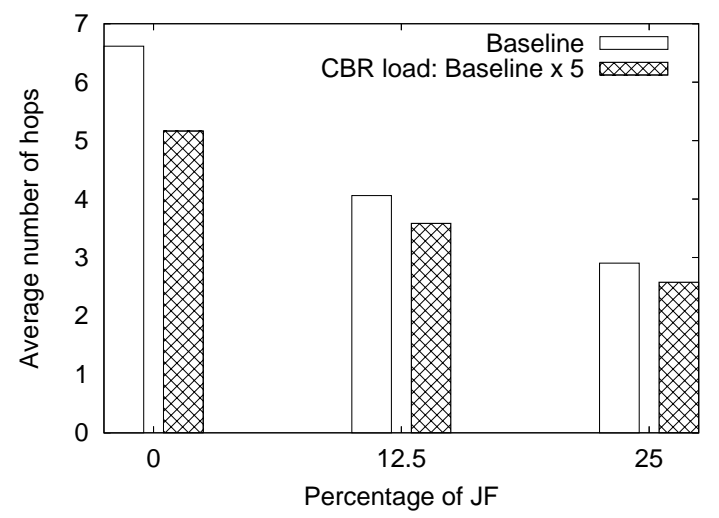

Figure 14: Hops for received packets with different CBR loads

Figure 14 illustrates this issue from an alternate perspective and depicts the average number of hops for a received packet under different loads. The figure shows that the presence of JF severely diminishes the allowed path lengths for successful communication in both the baseline and the overload cases.

\subsection{JellyFish Placement}

The baseline scenario considers grid placement as shown in Figure 7. Here we analyze the effect of different JF placement methods on the effectiveness of the JF attack and consider two additional methods: (i) random static placement in which JF are uniformly randomly placed within the geographical area, yet are non-mobile, and (ii) mobile JellyFish in which JF nodes have the same mobility characteristics as all other nodes.

Figure 15 shows the probability that an established route contains a JF node for the different placement techniques. From Section 3, we have that the probability of interception is given by $P_{\text {int }}=1-(1-a / N)^{h}$ for a fixed number of relay nodes $h$. 


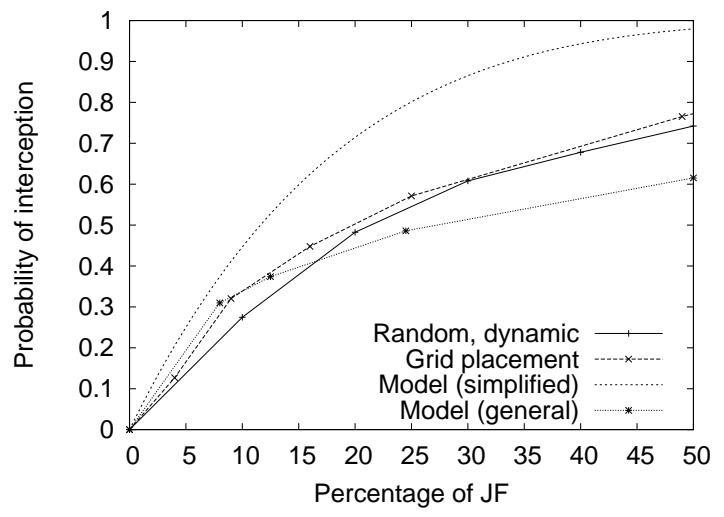

Figure 15: Probability of interception $P_{i n t}$ for different JF placement methods

As also described in Section 3, this expression is easily generalized to incorporate the hop count distribution via

$$
P_{\text {int }}=\sum_{h \geq 0}\left(1-(1-a / N)^{h}\right) \operatorname{Pr}(H=h)
$$

where $\operatorname{Pr}(H=h)$ is the probability of having $H=h$ relay nodes. The figure indicates that the simplified model which considers path length to be constant overestimates the number of intercepted flows. In contrast, by using the path length distribution as obtained from simulations together with Equation (6), the curve labeled "Model (general)" provides a close match with simulation.

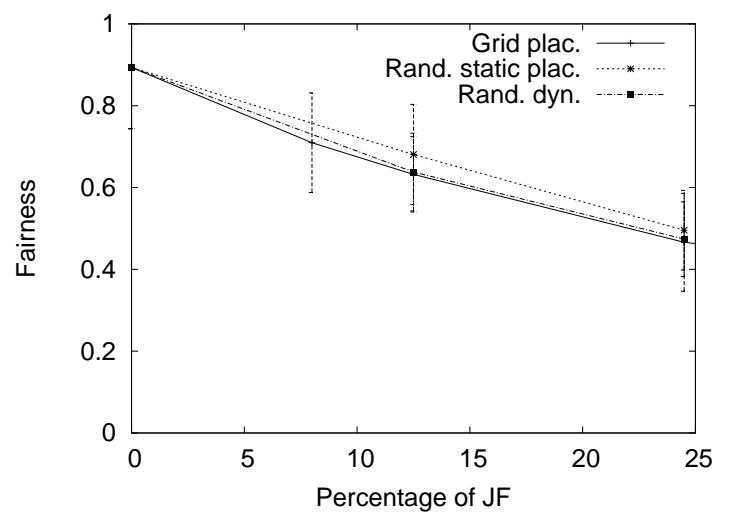

Figure 16: Fairness for different JF placement methods

Finally, Figure 16 indicates that grid placement and mobile JF are only slightly more harmful than random static placement. Although perhaps surprising that mobile and random placement is nearly as effective as grid placement's optimal coverage, the $2000 \mathrm{~m}$ $\times 2000 \mathrm{~m}$ topology together with $250 \mathrm{~m}$ receive range and 200 nodes imply that most of the considered area is covered with any of the placement techniques considered here, making them perform almost equally.

\subsection{Mobility}

The lifetime of a path is affected by the lifetime of the constituent relay nodes. That is, a path breaks when a single node on the path moves out of radio range of its upstream or downstream node. Thus, mobility is the key factor that controls path lifetime and we study this effect here.

Figure 17 depicts fairness for received packets for three mobility speeds, $1 \mathrm{~m} / \mathrm{s}, 10 \mathrm{~m} / \mathrm{s}$ and $20 \mathrm{~m} / \mathrm{s}$, with $10 \mathrm{~m} / \mathrm{s}$ representing the speed in the baseline case. Without attack, low mobility provides the best fairness as higher mobility hurts long-path-length flows in much the same way that JF hurt long-path-length flows. When there are $49 \mathrm{JF}$ in the system (24.5\%), the speed is of less importance, as all three speeds suffer high equivalent damage under attack.

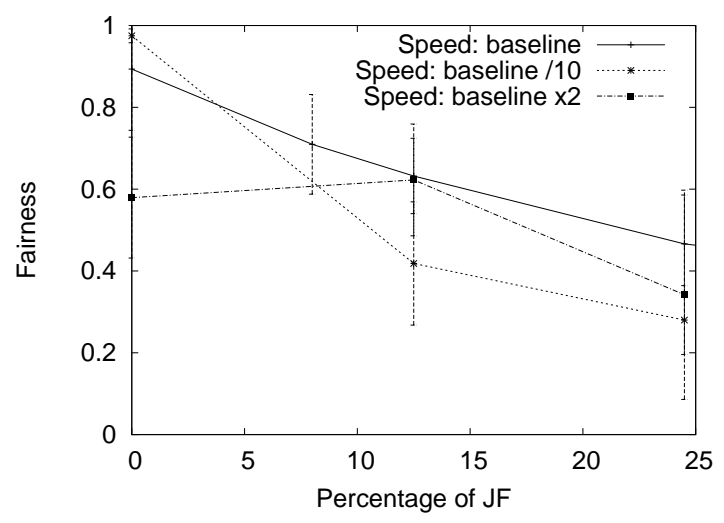

Figure 17: Fairness for different velocities

\subsection{Node Density}

In the baseline case, we consider a $2000 \mathrm{~m} \times 2000 \mathrm{~m}$ topology with 200 mobile nodes. Here we consider a scenario with $1 / 2$ the density in which 100 nodes are placed on a $2000 \mathrm{~m} \times 2000 \mathrm{~m}$ topology. Moreover, we consider a scenario with $1 / 6.25$ of the density in which 200 nodes are placed on a $5000 \mathrm{~m} \times 5000 \mathrm{~m}$ topology.

Figure 18 shows that for very low densities (baseline/6.25), the average number of hops is relatively low ( 4.7 hops) in spite of the large dimensions of the topology. In fact, due to the low density, the network is not fully connected such that long-range flows are unlikely to exist.

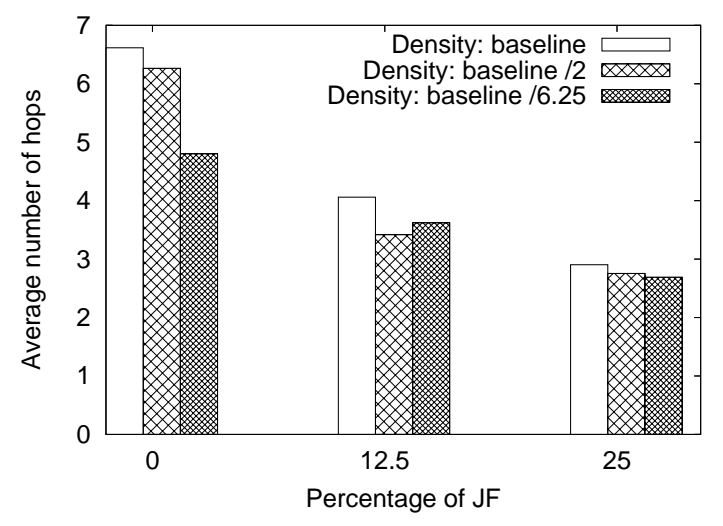

Figure 18: Hops for received packets for different densities

This partitioning is also visible from the fairness point of view as depicted in Figure 19. The figure indicates a significant decrease in fairness when the node density decreases to such low levels. 


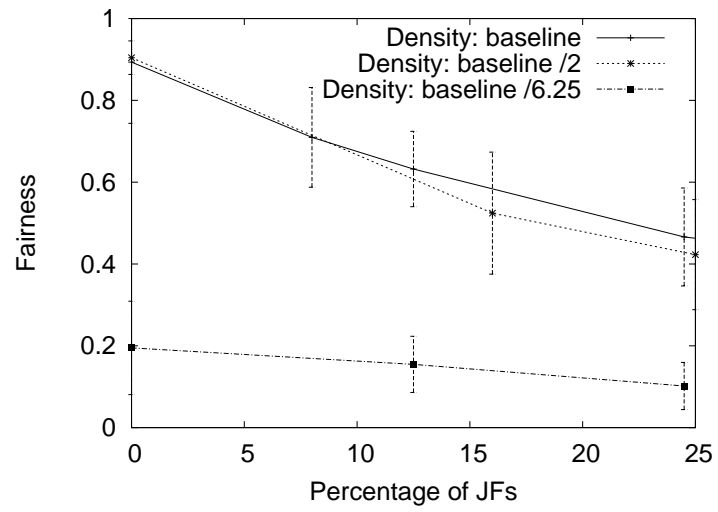

Figure 19: Fairness for different densities

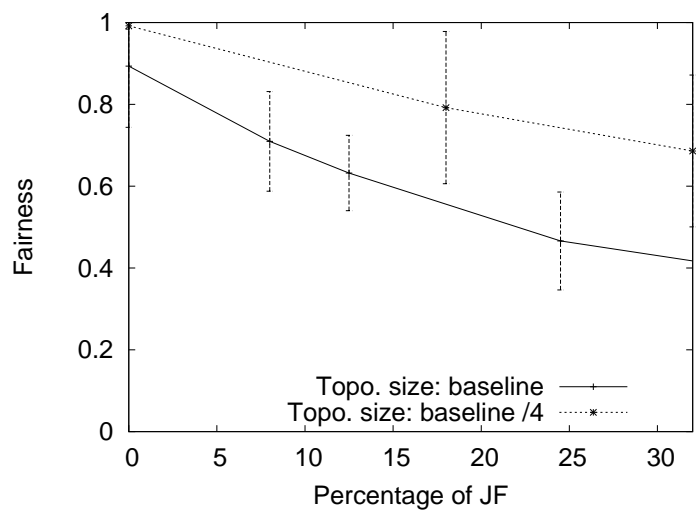

Figure 21: Fairness for different system sizes
With an increasing number of JF, system performance degrades similarly in the three cases. However, for the lowest density, the degradation is considerably less as fairness is already quite low even with no attackers.

\subsection{System Size}

Finally, we explore the effect of system size on attack scalability. In particular, as demonstrated in Section 3, the mean hop length plays a critical role in an attacks effectiveness. Here, we consider a $1000 \mathrm{~m} \times 1000 \mathrm{~m}$ system vs. the $2000 \mathrm{~m} \times 2000 \mathrm{~m}$ case of the baseline, and keep the node density constant resulting in 50 nodes.

Observe first from Figure 20 that without an attack, the mean hop length for a received packet is reduced by a factor of approximately 2 . Moreover, this factor is maintained across different percentages of JF as shown. Thus, the attack scalability remains unchanged with system size, yet the mean path length has a significant effect.

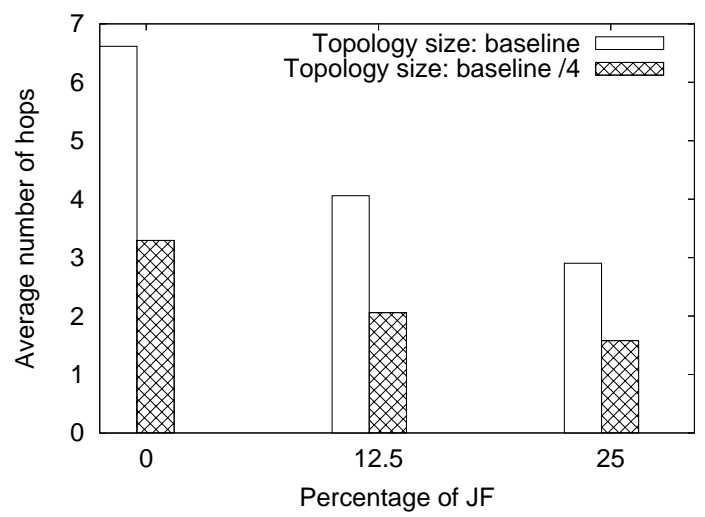

Figure 20: Hops for received packets for different system sizes

A similar trend is illustrated in Figure 21 which shows that a smaller system size results in higher initial fairness. That is, with shorter path lengths, flow throughputs are nearly identical. (Consider a small system in which all flows are within radio range: if the MAC protocol provides long term fairness, then the fairness index will be 1.) Yet, both system sizes obtain a similar scaling of a reduction in fairness with an increasing number of attackers.

\section{RELATED WORK}

Significant recent research efforts have focused on the challenge of securing mobile ad hoc networks with most work targeted towards securing routing protocols. Results can be classified according to the routing protocol(s) they consider and by the assumptions they make in terms of available security mechanisms (e.g., online/offline presence of an identity and key certification center, key distribution and revocation techniques, and cryptographic computation capabilities of the nodes). Other relevant parameters include the number of nodes, the mobility model, the underlying transmission protocols (MAC and physical layer), the propagation model of the radio channel, as well as the strength of the attacker (e.g., the number of controlled nodes).

In this section, we present an overview of related work in security in ad hoc networks with an emphasis on the mechanisms aiming at protecting against DoS attacks.

Securing Routing Protocols. The area which has attracted the most attention is security of the routing protocol and in particular, security of route establishment.

Ariadne [17], proposed by $\mathrm{Hu}$, Perrig and Johnson, protects source routing protocols such as DSR against a number of attacks. They propose a protocol to secure the routing discovery phase and to ensure that all forwarded packets follow the secure route. As they mentioned, this protocol does not protect the network against a legitimate but malicious relay node, which silently discards all or part of the packets. The two suggested counter-measures in [17] are Passive Acknowledgement and multi-path routing, both of which we discussed in Section 2 and the latter is also discussed below. Reference [17] also suggests blacklisting poorly performing nodes in order to prevent them from being included in future routes; we treat this issue below when discussing reputation-based systems.

In [17] Hu et al. also consider a route-request-flooding attack, which without counter-measures can be quite devastating, as each Route Request message generates a broadcast throughout the entire network. The proposed solution consists of having every node rate limit the Route Requests it is asked to relay. Although such a mechanism is indeed needed to protect the system from such attacks, we showed in Section 3 that such rate limiting can also delay a victim's ability to respond to an attack, and consequently will reduce the throughput of victims.

$\mathrm{Hu}$ et al. address the problem of securing distance vector protocols and have developed a protocol termed SEAD (Secure Efficient Ad hoc Distance vector routing protocol) [16]. In order to guard 
against several attacks including DoS attacks, SEAD makes use of one-way-hash chains and Merkle hash trees. The purpose of these structures is to authenticate the metric (distance to the target) and the sequence numbers (which are used in distance vector to assess the freshness of the information about a given route and, if not properly protected, could be exploited to mount attacks). They conclude that distance vector protocols are more difficult to secure than those based on source routing. In any case, we note that SEAD does not consider attacks against packet forwarding, nor does it address the use of multiple routes.

Other studied attacks include the Rushing Attack [20] (discussed in Section 3) and the Wormhole Attack [19]. Finally, [18] provides a description of four new mechanisms as tools for securing distance vector and path vector routing protocols; however, these mechanisms aim at protecting against attacks that are different from the those considered in this paper.

Finally, other proposals about secure routing protocols focus on secure route establishment and explicitly exclude packet dropping from their field of investigation [11,34]; we do not comment on them, as they are quite remote from our topic.

Usage of Multiple Routes. Papadimitratos and Haas developed the Secure Routing Protocol (SRP) [29] with a focus on using multiple routes. Unlike Ariadne, SRP relies exclusively on the mutual authentication of the end nodes (source and destination); hence, it does not require any authentication of the relay nodes, which makes the protocol more light-weight, but also more prone to attacks. For this reason, they have devised another, complementary protocol, termed SMT (Secure Message Transmission protocol) [30]. The principle of SMT is that the source and destination make use of an "Active Path Set" that consists of diverse, preferably disjoint paths that are initially deemed valid. The source disperses each outgoing message into a number of pieces. This operation introduces redundancy such that at the destination, a dispersed message can be successfully reconstructed, provided that sufficiently many pieces are received. Each dispersed message is transmitted across a different route and carries a Message Authentication Code, so that the destination can verify its integrity and the authenticity of its origin.

As selection of the optimal Active Path Set is an NP-complete problem, the Disjoint Pathset Selection Protocol is proposed to choose a set of reliable paths in nearly linear time [29]. An evaluation of the lifetime and of the size of the path set is provided via simulations. Although this approach is a promising building block to thwart DoS attacks, it has two limitations. The first, noted in [29], is the assumption that the MAC layer is implemented over channels that are well separated in time and frequency; in other words, there can be little correlation between transmissions from one node to its neighbors as a stronger correlation would certainly lead to a much smaller number of independent paths. The second limitation, related to the first one, is that the simulations do not include the effects of a MAC layer nor of TCP, both of which significantly impact performance in this scenario.

Securing Packet Forwarding. In order to mitigate attacks on packet forwarding, Marti et al. proposed Watchdog and Path Rater [27]. At the same time, Buttyan and Hubaux proposed the usage of a virtual currency, the nuglet [7]. More recently, Buchegger and Le Boudec devised the CONFIDANT reputation system [6], in which a node observes the behavior of its neighbors and updates its opinion about each of them accordingly. Michiardi and Molva proposed another reputation-based system, named CORE [28].

All such schemes rely on observations. Yet, as JellyFish are pro- tocol compliant, it will be difficult for neighbors to observe their misbehavior. Indeed, if attackers thwart PACK as described in Section 2.5, such neighbor-based detection is impossible. Alternatively, victims could share and cross correlate information about the nodes on poorly performing paths and eventually build confidence as to which nodes are JF. Although a time consuming and challenging task, one can presume that it can eventually be solved in many cases. In this context, our study should be viewed as characterizing the damage that JF can do until such a protocol can ensure that all $\mathrm{JF}$ are eliminated.

Finally, Jakobsson, Wetzel, and Yener describe a number of stealth attacks against ad hoc wireless networks [22]. This work includes the description of several relevant attacks, including DoS attacks, and suggests that a solution requires an appropriate reputation system. Although Jakobsson et al. present a thought-provoking overview of novel attacks, their work [22] does not contain quantitative results, as is the focus of our work.

Identification of the Attacking Node(s). We have considered that once a victim has detected a DoS attack, it will establish a new route. A more sophisticated reaction would also attempt to identify the attacking node(s) on the route exhibiting the anomalous behavior. For this purpose, Awerbuch et al. propose a technique aiming at identifying a "Byzantine node" on a given route [2]. The technique requires that the destination acknowledge every packet to the source; when the source detects that the number of lost packets is higher than a given threshold, it performs a binary search on the path in order to identify the faulty link. For that purpose, it polls specific nodes via probes and asks them to reply. The protocol targets having a malicious node be unable to distinguish between polling packets and normal ones, and be unable to know whether the source has started a probing session. Although a promising technique, this proposal has been investigated in static scenarios and its effectiveness with mobility is still unproven.

Other Techniques. Other researchers have studied packet forwarding from the context of incentive techniques. In this case, $\mathrm{ra}$ tional nodes attempt to maximize their benefit (e.g., their bitrate) of using the network; an example is SPRITE, by Zhong, Chen and Yang [36]. As such, these nodes do not aim at perpetrating DoS attacks.

All research efforts mentioned so far are focused on the network layer. The only study of Denial of Service at the MAC layer of ad hoc networks that we are aware of is by Gupta, Krishnamurthy and Faloutsos [15]. This work shows how vulnerable IEEE 802.11 can be to DoS attacks; it focuses more on the description of the attacks than on a way to thwart them.

A final topic, loosely related, is key establishment in ad hoc networks. Perhaps the first work in this area is by Zhou and Haas [37], but more recently, Luo et al. have proposed a scheme for access control in mobile ad hoc networks based on threshold cryptography [26]. Finally, Capkun, Hubaux and Buttyan have shown how mobility can be exploited to support key establishment [8]. These research efforts are quite remote from our topic, and we do not discuss them here.

\section{CONCLUSION}

In this paper, we studied a novel DoS attack perpetrated by JellyFish: relay nodes that stealthily misorder, delay, or periodically drop packets that they are expected to forward, in a way that leads astray end-to-end congestion control protocols. This attack is protocol-compliant and yet has a devastating impact on the throughput 
of closed-loop flows, such as TCP flows and congestion-controlled UDP flows. For completeness, we have also considered a wellknown attack, the Black Hole attack, as its impact on open-loop flows is similar to the effect of JellyFish on closed-loop flows.

We studied these attacks in a variety of settings and have provided a quantification of the damage they can inflict. We showed that, perhaps surprisingly, such attacks can actually increase the capacity of ad hoc networks as they will starve all multihop flows and provide all resources to one-hop flows that cannot be intercepted by JellyFish or Black Holes. As such a partitioned system is clearly undesirable, we also consider fairness measures and the mean number of hops for a received packet, as critical performance measures for a system under attack.

We assessed the effects of various performance factors (number of attacking nodes, mobility model, detection time, system size, etc.) on the above metrics via a simple analytical model and a substantial number of simulation experiments. In this way, we provide a quantitative study of the performance impact and scalability of DoS attacks in ad hoc networks.

Our objective is to provide guidelines for protocol designers who are developing DoS-resilience mechanisms: with a better understanding of the key attack factors and how to evaluate the impact of an attack, protocol designers can better determine if the overhead of deploying a counter-strategy is merited given the damage that an attack can inflict.

\section{REFERENCES}

[1] F. M. Anjum. TCP algorithms and multiple paths: Considerations for the future of the Internet. Information Systems Frontiers, 1:91-104, March 2004.

[2] Baruch Awerbuch, David Holmer, Cristina Nita-Rotaru, and Herbert Rubens. An on-demand secure routing protocol resilient to byzantine failures. In Proceedings of the ACM Workshop on Wireless Security (WiSe), Atlanta, Georgia, September 2002.

[3] E. Blanton and M. Allman. On making TCP more robust to packet reordering. ACM Computer Communications Review, 32(1):20-30, January 2003.

[4] S. Bohacek, J. Hespanha, J. Lee, C. Lim, and K. Obraczka. TCP-PR: TCP for persistent packet reordering. In Proceedings of the 23rd IEEE International Conference on Distributed Computing Systems, May 2003.

[5] L. Brakmo, S. O'Malley, and L. Peterson. TCP Vegas: New techniques for congestion detection and avoidance. In Proceedings of ACM SIGCOMM '94, May 1994.

[6] Sonja Buchegger and Jean-Yves Le Boudec. Performance Analysis of the CONFIDANT Protocol: Cooperation Of Nodes - Fairness In Dynamic Ad-hoc NeTworks. In Proceedings of IEEE/ACM Symposium on Mobile Ad Hoc Networking and Computing (MobiHoc), Lausanne, CH, June 2002.

[7] L. Buttyan and J. P. Hubaux. Enforcing Service Availability in Mobile Ad-Hoc WANs. In Proceedings of IEEE/ACM Workshop on Mobile Ad Hoc Networking and Computing (MobiHoc), Boston, MA, USA, August 2000.

[8] S. Capkun, J. P. Hubaux, and L. Buttyan. Mobility Helps Peer-to-Peer Security. To appear in IEEE Transactions on Mobile Computing, 2005.

[9] C. Casetti, M. Gerla, S. Mascolo, M. Sanadidi, and R. Wang. TCP Westwood: Bandwidth estimation for enhanced transport over wireless links. In Proceedings of ACM MobiCom 2001, Rome, Italy, July 2001.
[10] M. Chan and R. Ramjee. TCP/IP performance over 3G wireless links with rate and delay variation. In Proceedings of ACM MobiCom 2002, Atlanta, GA, October 2002.

[11] Bridget Dahill, Kimaya Sanzgiri, Brian Neil Levine, Clay Shields, and Elizabeth M. Belding-Royer. A secure routing protocol for ad hoc networks. In Proceedings of ICNP, 2002.

[12] K. Fall and S. Floyd. Simulation-based comparison of Tahoe, Reno and SACK TCP. ACM Computer Communications Review, 5(3):5-21, July 1996.

[13] S. Floyd, M. Handley, J. Padhye, and J. Widmer. Equation-based congestion control for unicast applications. In Proceedings of ACM SIGCOMM 'OO, Stockholm, Sweden, August 2000.

[14] M. Gerla, S. Lee, and G. Pau. TCP Westwood simulation studies in multiple-path cases. In Proceedings of SPECTS 2002, San Deigo, CA, July 2002.

[15] V. Gupta, S.V. Krishnamurthy, and M. Faloutsos. Denial of Service Attacks at the MAC Layer in Wireless Ad Hoc Networks. In Proceedings of MILCOM, 2002.

[16] Yih-Chun Hu, David B. Johnson, and Adrian Perrig. SEAD: Secure Efficient Distance Vector Routing for Mobile Wireless Ad Hoc Networks. Ad Hoc Networks, 1(1):175-192, 2003.

[17] Yih-Chun Hu, Adrian Perrig, and David B. Johnson. Ariadne: A secure on-demand routing protocol for ad hoc networks. In Proceedings of the Eighth ACM International Conference on Mobile Computing and Networking (MobiCom 2002), September 2002.

[18] Yih-Chun Hu, Adrian Perrig, and David B. Johnson. Efficient security mechanisms for routing protocols. In Network and Distributed System Security Symposium, NDSS '03, February 2003.

[19] Yih-Chun Hu, Adrian Perrig, and David B. Johnson. Packet leashes: A defense against wormhole attacks in wireless networks. In Proceedings of IEEE Infocom 2003, April 2003.

[20] Yih-Chun Hu, Adrian Perrig, and David B. Johnson. Rushing attacks and defense in wireless ad hoc network routing protocols. In Proceedings of WiSe 2003, September 2003.

[21] R. Jain. The Art of Computer System Performance Analysis. John Wiley and Sons, Inc., 1991.

[22] M. Jakobsson, S. Wetzel, and B. Yener. Stealth attacks on ad hoc wireless networks. In Proceedings of VTC, 2003.

[23] David B. Johnson and D. Maltz. The dynamic source routing protocol for mobile ad hoc networks (DSR), April 2003. http://www.ietf.org/internet-drafts/draft-ietf-manet-dsr09.txt.

[24] V. Kawadia and P. R. Kumar. Power control and clustering in ad hoc networks. In Proceedings of IEEE Infocom, 2003.

[25] A. Kuzmanovic and E. Knightly. Low-Rate TCP-Targeted Denial of Service Attacks (The Shrew vs. the Mice and Elephants. In Proceedings of ACM SIGCOMM 2003, Karlsruhe, Germany, August 2003.

[26] Haiyun Luo, Jiejun Kong, Petros Zerfos, Songwu Lu, and Lixia Zhang. URSA: Ubiquitous and Robust Access Control for Mobile Ad-Hoc Networks. To appear in IEEE/ACM Transactions on Networking. October 2004.

[27] Sergio Marti, T. J. Giuli, Kevin Lai, and Mary Baker. Mitigating routing misbehavior in mobile ad hoc networks. In Mobile Computing and Networking, pages 255-265, 2000.

[28] P. Michiardi and R. Molva. CORE: A Collaborative Reputation Mechanism To Enforce Node Cooperation In 
Mobile Ad Hoc Networks. In Proceedings of The 6th IFIP Communications and Multimedia Security Conference, Portorosz, Slovenia, September 2002.

[29] P. Papadimitratos and Z. Haas. Secure routing for mobile ad hoc networks. In Proceedings of CNDS, 2002.

[30] P. Papadimitratos and Z. Haas. Secure data transmission in mobile ad hoc networks. In Proceedings of WiSe, 2003.

[31] V. Paxson and M. Allman. Computing TCP's retransmission timer, November 2000. Internet RFC 2988.

[32] N. Sadagopan, F. Bai, B. Krishnamachari, and A. Helmy. PATHS: analysis of path duration Statistics and their impact on reactive MANET routing protocols. In Proceedings of MobiHoc, 2003.

[33] F. Wang and Y. Zhang. Improving TCP performance over mobile ad-hoc networks with out-of-order detection and response. In Proceedings of IEEE/ACM MobiHoc, Lausanne, $\mathrm{CH}$, June 2002.

[34] Manuel Zapata and N. Asokan. Securing ad hoc routing protocols. In Proceedings of the ACM Workshop on Wireless Security (WiSe), Atlanta, Georgia, September 2002.

[35] M. Zhang, B. Karp, S. Floyd, and L. Peterson. RR-TCP: A reordering robust TCP with DSACK. In Proceedings of IEEE ICNP 2003, Atlanta, GA, November 2003.

[36] Sheng Zhong, Jiang Chen, and Yang Richard Yang. Sprite: A simple, cheat-proof, credit-based system for mobile ad-hoc networks. In Proceedings of IEEE Infocom, 2003.

[37] Lidong Zhou and Zygmunt J. Haas. Securing ad hoc networks. IEEE Network, 13(6), 1999. 